\title{
EXTREMAL PLANE QUASICONFORMAL MAPPINGS WITH GIVEN BOUNDARY VALUES
}

\author{
BY EDGAR REICH AND KURT STREBEL \\ Communicated by F. W. Gehring, October 23, 1972
}

1. Introduction. Let $\Omega_{i}, i=1,2$, be regions in the complex plane, $f(z)$ a quasiconformal mapping of $\Omega_{1}$ onto $\Omega_{2}$. Let $Q_{f}$ denote the class of all quasiconformal mappings of $\Omega_{1}$ onto $\Omega_{2}$ which have the same boundary values as $f$. A mapping $f^{*} \in Q_{f}$ will be called extremal for its boundary values if it is $K^{*}$-quasiconformal and if there exists no $K$-quasiconformal mapping in $Q_{f}$ with $K<K^{*}$. The quantity $K^{*}=K^{*}(f)$ is the extremal dilatation for the class $Q_{f}$. (As is well known [3], there may be more than one $K^{*}$-quasiconformal mapping in the class $Q_{f}$.) In the present account, which is only an abstract, we restrict ourselves to the case $\Omega_{1}=\Omega_{2}=E$ $=\{|z|<1\}$. Generalizations to Riemann surfaces will be referred to in a detailed account, giving proofs, further results, and applications that is to appear elsewhere.

In what follows, the $L^{1}$ norm $\iint_{E}|\varphi(z)| d x d y$ of functions $\varphi(z)$ holomorphic in $E$ will be denoted by $\|\varphi\|$.

In 1969, R. S. Hamilton [1] proved the following: If $f^{*} \in Q_{f}$ is an extremal mapping, $\kappa^{*}(z)=f_{\frac{z}{z}}^{*} / f_{z}^{*}$, then

$$
\sup _{\|\varphi\| \leqq 1}\left|\iint_{E} \kappa^{*}(z) \varphi(z) d x d y\right|=k^{*}(f)=\frac{K^{*}(f)-1}{K^{*}(f)+1}
$$

A central result of the present work is (\$3) that condition (1.1) characterizes extremal mappings of $E$.

2. Estimates for $K^{*}(f)$. The following is a generalization of an inequality proved in [2] from the case $K^{*}(f)=1$ to arbitrary $K^{*}(f)$.

THEOREM 2.1. If $f(z)$ is a quasiconformal self-mapping of $E, \kappa(z)=f_{\bar{z}} / f_{z}$, and if $\varphi(z)$ is holomorphic in $E$, then

$$
\begin{aligned}
\left|\int_{E} \frac{\kappa(z)}{1-|\kappa(z)|^{2}} \varphi(z) d x d y\right| \leqq & \frac{k^{*}(f)}{1+k^{*}(f)}\|\varphi\| \\
& +\iint_{E} \frac{|\kappa(z)|^{2}}{1-|\kappa(z)|^{2}}|\varphi(z)| d x d y .
\end{aligned}
$$

AMS (MOS) subject classifications (1970). Primary 30A60.

${ }^{1}$ Supported by GP 27275. 
For certain special holomorphic functions $\varphi(z)$ the quantity on the left side of (2.1) can be estimated in a useful way from below. Let us select $n$ equally spaced points on $\{|z|=1\}, n \geqq 4$. These are mapped by $w=f(z)$ onto $n$ points of $\{|w|=1\}$. The unique extremal quasiconformal mapping of $E$ onto $E$, which preserves this correspondence of $n$-gons, is [4] a Teichmüller mapping with complex dilatation

$$
\left.k_{n}\left(\overline{\varphi_{n}(z)}\right) /\left|\varphi_{n}(z)\right|\right),
$$

where $\varphi_{n}(z)$ is holomorphic in $E$ and has finite norm $\left\|\varphi_{n}\right\|$. Without loss of generality we can assume that $\left\|\varphi_{n}\right\|=1$. It is then possible to prove the following:

THEOREM 2.2. Let $f(z)$ be a quasiconformal self mapping of $E, \kappa(z)=f_{\bar{z}} / f_{z}$. Let $n \geqq 4$ points be selected on $\partial E$ and $k_{n}$ and $\varphi_{n}(z)$ determined as above. Then

$$
\left|\iint_{E} \frac{\kappa(z)}{1-|\kappa(z)|^{2}} \varphi_{n}(z) d x d y\right| \geqq \frac{k_{n}}{1-k_{n}}-\iint_{E} \frac{|\kappa(z)|^{2}}{1-|\kappa(z)|^{2}}\left|\varphi_{n}(z)\right| d x d y .
$$

Given $f$, let us introduce the quantities

$$
\begin{aligned}
I(f) & =\sup _{\|\varphi\| \leqq 1}\left|\iint_{E} \frac{\kappa(z) \varphi(z)}{1-|\kappa(z)|^{2}} d x d y\right|\left(\kappa(z)=f_{\bar{z}} / f_{z}\right), \\
\Delta(f) & =\sup _{\|\varphi\| \leqq 1} \iint_{E} \frac{|\kappa(z)|^{2}|\varphi(z)|}{1-|\kappa(z)|^{2}} d x d y .
\end{aligned}
$$

The following then follows easily as a corollary of Theorems 2.1 and 2.2:

THEOREM 2.3. The maximal dilatation $K^{*}(f)$ which is extremal for the class $Q_{f}$ satisfies the estimate

$$
\frac{1}{1-2 I(f)+2 \Delta(f)} \leqq K^{*}(f) \leqq 1+2 I(f)+2 \Delta(f) .
$$

REMARK. When $f$ is a Teichmüller mapping with finite norm both inequalities become equalities.

3. Characterization of complex dilatations of extremal mappings. Let

$$
H(f)=\sup _{\|\varphi\| \leqq 1}\left|\iint_{E} \kappa(z) \varphi(z) d x d y\right| .
$$

By means of Theorem 2.2 one obtains a new proof of Hamilton's necessary condition (1.1), with an explicit identification of an extremal sequence for $H(f)$ :

THEOREM 3.1. If $f^{*} \in Q_{f}$ is extremal, $\kappa^{*}(z)=f_{\frac{z}{z}}^{*} / f_{z}^{*}$, then 


$$
\lim _{n \rightarrow \infty}\left|\iint_{E} \kappa^{*}(z) \varphi_{n}(z) d x d y\right|=H(f)=k^{*}(f) .
$$

Furthermore, as a deduction from Theorem 2.3, the converse also follows:

THEOREM 3.2. A necessary and sufficient condition for the quasiconformal mapping $f$ of $E$ onto $E$ to be extremal for its boundary values is that

$$
H(f)=\underset{z \in E}{\operatorname{ess} \sup }|\kappa(z)| .
$$

\section{REFERENCES}

1. Richard S. Hamilton, Extremal quasiconformal mappings with prescribed boundary values, Trans. Amer. Math. Soc. 138 (1969), 399-406.

2. Edgar Reich and Kurt Strebel, On quasiconformal mappings which keep the boundary points fixed, Trans. Amer. Math. Soc. 138 (1969), 211-222. MR 38 \# 6059.

3. Kurt Strebel, Zur Frage der Eindeutigkeit extremaler quasikonformer Abbildungen des Einheitskreises. I, II, Comment. Math. Helv. 36 (1962), 306-323; ibid. 39 (1964), 77-89. MR 31 \#346.

4. 1 Über quadratische Differentiale mit geschlossenen Trajektorien und extremale quasikonforme Abbildungen, Festband zum 70, Geburtstag von R. Nevanlinna, Springer, Berlin, 1966, pp. 105-127.

School of Mathematics, University of Minnesota, Minneapolis, Minnesota 55455

MATHEMATISChes INSTItUT, UNIVERSITÄT ZÜRICH, CH-8006 ZÜRICH/ZH, SwITZERLAND 